\title{
Systemic lupus erythematosus in 50 year olds
}

\author{
I. Domenech, O. Aydintug, R. Cervera, M. Khamashta, A. Jedryka-Goral, \\ J.L. Vianna and G.R.V. Hughes
}

Lupus Arthritis Research Unit, The Rayne Institute, St Thomas' Hospital, London, SE1 7HE

\begin{abstract}
Summary: We compared the clinical and serological characteristics of 15 patients with onset of systemic lupus erythematosus after the age of $\mathbf{5 0}$ with those of 232 younger patients. The sex distribution was similar in both groups. All 15 patients were Caucasian. Autoimmune thyroiditis was found in $20 \%$ of the elderly patients. Initial manifestations, which presented more frequently in the older group, included thrombocytopenia $(P<0.05)$, sicca syndrome $(P<0.01)$ and cardiomyopathy $(P<0.005)$, whereas butterfly rash $(P<0.05)$ presented more frequently in the younger group. Analysis of cumulative clinical symptoms showed that butterfly rash $(P<0.05)$ and livedo reticularis $(P<0.05)$ were less frequent in the elderly. However, this group presented a significantly increased incidence of sicca syndrome $(P<0.005)$ and cardiomyopathy $(P<0.005)$. Antibodies to double-stranded DNA tended to occur less frequently in older patients $(P<0.05)$.
\end{abstract}

\section{Introduction}

Systemic lupus erythematosus (SLE) is predominantly an autoimmune disease of young women, its peak onset being between 15 and 40 years of age. ${ }^{1}$ SLE diagnoses have increased and it seems sensible to attribute this to improved diagnostic tests and the recognition of mild disease manifestations. ${ }^{2}$ With this increased awareness has come an increased recognition of lupus and lupus-like disease over a wider age span.

There have been several studies dealing with late-onset SLE, suggesting that age at onset modifies the clinical expression of the disease in terms of onset, clinical presentation, pattern of organ involvement and serological findings. ${ }^{3-10}$ Disease onset in elderly patients seems to be more insidious than in younger patients. ${ }^{3,8,10}$ Different patterns of presentation have been described including polymyalgia rheumatica-type syndrome, ${ }^{6}$ lung disease 5,6 and arthritis. ${ }^{3,8}$ The most consistent feature in elderly patients has been the higher frequency of serositis ${ }^{3,7}$ and sicca syndrome ${ }^{5,6}$ and a lower frequency of Raynaud's, ${ }^{9}$ neuropsychiatric involvement, ${ }^{6}$ serious renal disease ${ }^{3}$ and butterfly rash. $6,10,11$ The presence of serological abnormalities has been reported to be different in elderly patients. ${ }^{3-6,10}$

\footnotetext{
Correspondence: M. Khamashta, M.D.

Grant support: Dr I. Domenech is a Research Fellow sponsored by Spanish Fondo Investigaciones de la Seguridad Social. This study was supported by grants from the Lupus UK and Jean Shanks Foundation. Accepted: 10 December 1991
}

Previous reports used different age limits and definition of age at onset, but most of them used the arbitrary division at the age of 50 separating the two populations of older-onset and younger on set. ${ }^{3,7,10-12}$ In order to define better the clinical an immunological features with late onset SLE, we analyse a series of 247 unselected SLE patients.

\section{Patients and methods}

Our study comprised 247 patients with SLE who have been seen consecutively in the Lupus Clinic at St Thomas' Hospital, London either as in-patients or outpatients between 1985 and 1991. All met the American Rheumatism Association revised classification criteria for SLE. ${ }^{13}$ In 15 patients the onset of disease occurred after the age of 50 (older-onset group) and in the remaining 232 patients, the onset was before the age of 50 (younger-onset group). Age at onset was defined as patient age when the first disease manifestation clearly attributable to SLE occurred. Arbitrary limits of 50 years at onset of disease were adopted.

A clinical and laboratory assessment according to a pre-established protocol was performed. Associated and familial autoimmune disease, symptoms at onset, cumulative clinical features, treatment and laboratory test results were collected during this period. Clinical features were defined as: (1) cutaneous involvement - butterfly rash, discoid lupus and photosensitivity; (2) arthritis joint tenderness, swelling and/or pain on motion; (3) serositis - including pleuritis, pericarditis or both; (4) nephropathy - abnormal urine sediment, 
proteinuria $>500 \mathrm{mg} /$ day or otherwise unexplained elevation of serum creatinine $>115 \mu \mathrm{mol} / \mathrm{l}$; (5) neurological involvement - psychosis, seizure or objective neurological findings in the absence of another definable cause; (6) cardiac involvement cardiomyopathy, heart valve dysfunction assessed by echocardiography; (7) other clinical features, including Raynaud's syndrome, livedo reticularis, myositis, thrombosis and lung disease.

Laboratory findings analysed included occurrence of antinuclear antibody (ANA), antibodies to double-stranded DNA (anti-dsDNA), extractable nuclear antigen (ENA) and complement fractions (C3, C4). ANA was determined by indirect immunofluorescence using mouse liver as substrate. Anti-dsDNA antibodies as detected by the crithidia lucillae immunofluorescent method as screening, and Farr's ammonium sulphate precipitation technique for confirmation. ENA was detected by counterimmunoelectrophoresis using rabbit thymus and human spleen extract. All patients were tested for the presence of anticardiolipin antibodies and lupus anticoagulant activity. The anticardiolipin antibodies were measured by a standardized ELISA method, as described by Gharavi et al. ${ }^{14}$ Lupus anticoagulant activity was detected by the Exner's method. ${ }^{15}$

\section{Data analysis}

The frequency of clinical features was compared in younger and older patients by chi-square analysis and Yates' correction test for analysing qualitative differences. In quantitative variables, means between two groups were compared using the paired two-tailed $t$-test and Mann-Whitney when applicable. A $P$ value of 0.05 or less was considered statistically significant.

\section{Results}

\section{Patients}

Figure 1 shows the age of onset of our 247 SLE patients. In 15 patients $(6.1 \%)$, the onset of disease occurred after the age of 50 . Their mean age at onset was 56.5 years (range $50-70$ years) compared with 26.4 years (range $1-49$ years) for the youngeronset patients. The percentage of female was similar in both groups, 14 of $15(93.3 \%)$ in the older-onset group and 217 of $232(93.5 \%)$ in the younger-onset group. All 15 patients were Caucasian, although $18(9.2 \%)$ of our total population were black.

In relation to other autoimmune disease associations, three of our 15 elderly patients $(20 \%)$ had associated autoimmune thyroiditis, as defined by antithyroid antibodies and thyroid hormonal dis-

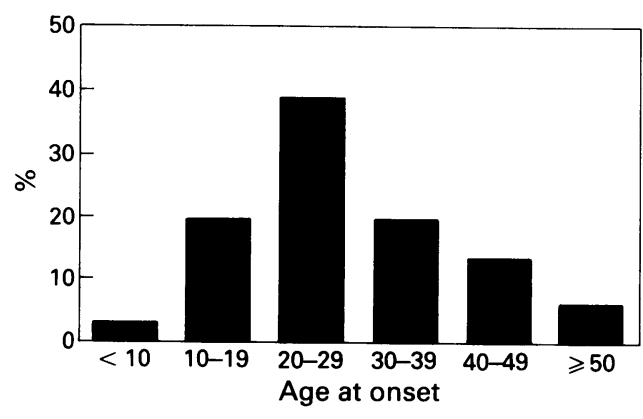

Figure 1 Age at onset in 247 patients with SLE.

orders. In contrast, only eight of our 232 younger patients $(3.4 \%)$ had associated autoimmune thyroiditis $(P<0.002)$. Familial association with autoimmune disease was not statistically significant.

\section{Clinical features at onset}

The clinical features at onset in the two groups are shown in Table I. Thrombocytopenia $(P<0.05)$, sicca syndrome $(P<0.01)$ and cardiomyopathy $(P<0.005)$ were more common initial manifestations in older patients than in the younger ones, whereas butterfly rash $(P<0.05)$ was more frequent in the younger group. In the older-onset group, arthritis was the most common manifestation at the onset, presenting in eight of 15 patients $(53 \%)$. Renal involvement, livedo reticularis and myositis were only seen as initial symptoms in the younger group.

\section{Analysis of cumulative clinical symptoms}

Analysis of cumulative clinical symptoms showed that butterfly rash $(P<0.05)$ and livedo reticularis $(P<0.05)$ were less frequent in the elderly. However, this group presented a significantly increased incidence of sicca syndrome $(P<0.005)$ and cardiomyopathy $(P<0.005)$ (Table II). No differences between the groups were found in the frequency of other clinical features including serositis, nephropathy, neurological or pulmonary involvement. Arthritis was the most common manifestation in the elderly patients $(80 \%)$, as in all our patients.

Therapy employed in the two groups did not show any statistically significant differences but all 32 patients who had received cyclophosphamide pulses were included in the younger-onset group (data not shown). 
Table I Initial symptoms of disease of elderly compared to younger patients

\begin{tabular}{lrlc}
\hline Manifestations & $\begin{array}{c}\text { Younger onset } \\
(\mathrm{n}=232)\end{array}$ & $\begin{array}{c}\text { Older onset } \\
(\mathrm{n}=15)\end{array}$ & $\mathrm{P}$ \\
\hline Butterfly rash & $93(40 \%)$ & $2(13 \%)$ & $<0.05$ \\
Discoid lupus & $35(15 \%)$ & 0 & $\mathrm{NS}$ \\
Photosensitivity & $49(21 \%)$ & $2(13 \%)$ & NS \\
Arthritis & $144(62 \%)$ & $8(53 \%)$ & NS \\
Serositis & $33(14 \%)$ & $2(13 \%)$ & NS \\
Nephropathy & $20(9 \%)$ & 0 & NS \\
Neuropsychiatric & $29(13 \%)$ & $3(20 \%)$ & NS \\
Thrombocytopenia & $12(5 \%)$ & $3(20 \%)$ & $<0.05$ \\
Sicca syndrome & $10(4 \%)$ & $3(20 \%)$ & $<0.01$ \\
Raynaud's phenomena & $42(18 \%)$ & $2(13 \%)$ & NS \\
Livedo reticularis & $7(3 \%)$ & 0 & NS \\
Myositis & $8(3 \%)$ & 0 & NS \\
Cardiomyopathy & $2(1 \%)$ & $2(13 \%)$ & $<0.005$ \\
Lung involvement & $5(2 \%)$ & $1(7 \%)$ & NS \\
\hline
\end{tabular}

NS $=$ not significant .

Table II Cumulative clinical manifestations of SLE related to age at onset

\begin{tabular}{lccc}
\hline Manifestations & $\begin{array}{c}\text { Younger onset } \\
(\mathrm{n}=232)\end{array}$ & $\begin{array}{c}\text { Older onset } \\
(\mathrm{n}=15)\end{array}$ & $\mathrm{P}$ \\
\hline Butterfly rash & $137(51 \%)$ & $4(27 \%)$ & $<0.02$ \\
Discoid lupus & $57(25 \%)$ & $2(13 \%)$ & NS \\
Photosensitivity & $91(39 \%)$ & $4(27 \%)$ & NS \\
Arthritis & $195(84 \%)$ & $12(80 \%)$ & NS \\
Serositis & $92(40 \%)$ & $7(47 \%)$ & NS \\
Nephropathy & $71(31 \%)$ & $3(20 \%)$ & NS \\
Neuropsychiatric & $78(34 \%)$ & $6(40 \%)$ & NS \\
Sicca syndrome & $49(21 \%)$ & $9(60 \%)$ & $<0.005$ \\
Raynaud's phenomena & $116(50 \%)$ & $6(40 \%)$ & NS \\
Livedo reticularis & $37(3 \%)$ & 0 & $<0.05$ \\
Myositis & $24(10 \%)$ & 0 & NS \\
Cardiomyopathy & $4(2 \%)$ & $2(13 \%)$ & $<0.005$ \\
Heart valve disease & $18(8 \%)$ & $1(7 \%)$ & NS \\
Thrombosis & $55(24 \%)$ & $1(7 \%)$ & NS \\
Lung involvement & $5(2 \%)$ & $1(7 \%)$ & NS \\
\hline
\end{tabular}

$\mathrm{NS}=$ not significant

\section{Serological findings (Table III)}

The frequency of detection of antibodies to doublestranded DNA was lower in older patients $(P<0.05)$. IgM anticardiolipin antibodies were detected in greater proportion in the elderly group $(P<0.05)$. The frequency of detection of serum complement fractions (C3, C4), IgG anticardiolipin antibodies, and ANA were similar in both groups. Ro antibodies tended to occur more frequently in the elderly, but this was not statistically significant.

\section{Discussion}

SLE is not usually thought of as a disease of the elderly, but recently, several reports have been calling for greater consideration of this group. ${ }^{3-11}$ The purpose of the present study was to analyse the presenting manifestations, the patterns of organ involvement and the laboratory findings in this subset of SLE patients. Fifteen $(6.1 \%)$ of 247 patients with SLE developed the disease after the age of 50. This frequency is slightly lower than 
Table III Serological findings in older and younger onset SLE patients

\begin{tabular}{lccc}
\hline & $\begin{array}{c}\text { Younger-onset } \\
(\mathrm{n}=232)\end{array}$ & $\begin{array}{c}\text { Older-onset } \\
(\mathrm{n}=15)\end{array}$ & $\mathrm{P}$ \\
\hline ANA & $181(78 \%)$ & $10(67 \%)$ & NS \\
Anti-dsDNA & $142(61 \%)$ & $5(33 \%)$ & $<0.05$ \\
Low C3 & $35(15 \%)$ & 0 & NS \\
Low C4 & $56(24 \%)$ & $2(13 \%)$ & NS \\
IgM aCL & $15(6 \%)$ & $3(20 \%)$ & $<0.05$ \\
IgG aCL & $34(15 \%)$ & $2(13 \%)$ & NS \\
Ro (SSA) & $75(32 \%)$ & $2(13 \%)$ & NS \\
La (SSB) & $39(17 \%)$ & $2(13 \%)$ & NS \\
RNP & $37(16 \%)$ & $1(7 \%)$ & NS \\
Sm & $21(9 \%)$ & 0 & NS \\
Lupus anticoagulant & $22(10 \%)$ & $1(7 \%)$ & NS \\
\hline
\end{tabular}

IgM $\mathrm{aCL}=\mathrm{IgM}$ anticardiolipin antibodies; $\mathrm{IgG} \mathrm{aCL}=\mathrm{IgG}$ anticardiolipin antibodies; $\mathrm{NS}=$ not significant.

reported in other series $(7-18 \%),{ }^{4,5,9,10}$ which could be due to the fact that our clinic is predominantly an outpatient clinic. Although some reports have suggested that the female predominance is not so marked in the elderly, ${ }^{4,10,12}$ our series as well as others reported in the literature ${ }^{5,6,9}$ have found similar female predominance. All of our olderonset group were Caucasian, in accordance with previous reports ${ }^{4,8}$ which have concluded that younger, though not older, black females are particularly predisposed to SLE. Furthermore, our finding of a high incidence of autoimmune thyroiditis in the elderly group studied is supported by a recent study demonstrating a similar high prevalence of autoimmune thyroid disease in an older SLE population. ${ }^{16}$

There is a general agreement that age modifies the clinical features of disease, but the different clinical descriptions of SLE in elderly patients are conflicting. In our series, butterfly rash is a less frequent manifestation at the onset in elderly patients as has been reported in other series. ${ }^{7,9,11}$ Most common patterns of onset were sicca syndrome, thrombocytopenia and arthritis. Obviously alternative working diagnoses could be Sjogren's syndrome, idiopathic thrombocytopenic purpura and rheumatoid arthritis, respectively. ${ }^{5,8}$ The pattern of organ involvement included an increased frequency of sicca syndrome and cardiomyopathy in the elderly group. The increased frequency of sicca syndrome has been reported by other authors. ${ }^{5-7}$ Butterfly rash and livedo reticularis were less frequent in elderly patients in our series. Similar results were found by other authors. ${ }^{7,9} \mathrm{We}$ did not find differences in renal, neurological and lung involvement between both groups.

Assessment of therapy employed during the course of disease revealed no significant differences between the two groups, but all patients treated with cyclophosphamide pulses were in the youngeronset group, although this was not statistically significant. Other authors found that the elderly group was treated with lower doses of corticosteroids. ${ }^{9}$

Age, also, influences the serological manifestations of SLE. Anti-dsDNA antibodies tended to occur less frequently in older patients in our series as reported previously. ${ }^{9,10}$ Wilson et al. ${ }^{3}$ found fewer anti-DNA antibodies and a less nephritis with age. Font et al. ${ }^{10}$ and Manussakis et al. ${ }^{17}$ found anticardiolipin antibodies were present in higher proportion in the elderly population. Our own data showed only modest increase in IgM anticardiolipin antibody frequency. A high frequency of antibody to the cytoplasmic antigen Ro (SSA) has also been reported, ${ }^{5,12}$ and in our series, this antibody occurred more frequently in the olderonset group, although not to a statistically significant degree.

Although SLE is typically a disease of young adults, clinicians should note that in the future it may be diagnosed more frequently among older adults. Apart from the two different patterns of presentation in older patients, including a polymyalgia-like ${ }^{6}$ and rheumatoid arthritis-like picture, ${ }^{8}$ our patients presented with thrombocytopenia as the first manifestation. SLE diagnosis in elderly patients could prove difficult because atypical signs and symptoms in this age group are very common and could delay the diagnosis. ${ }^{18}$ Late onset SLE presents a major overlap with Sjogren syndrome, and it is often difficult to make a differential diagnosis. ${ }^{19}$ Elderly SLE patients behave like Sjogren syndrome patients, and their high frequency of Ro antibodies could help to explain the milder disease course that some authors noted in 
this population. ${ }^{3,5}$ However, thrombocytopenia may be considered an independent risk factor for a worse prognosis in SLE, ${ }^{19}$ and recent studies do not demonstrate better survival rates for this population. 6,20

It seems clear that age modifies the clinical expression of the disease. This difference could be related to different genetic determinants of disease expression $^{12}$ and/or environmental factors. ${ }^{6}$ Furthermore, the less exuberant expression of SLE in older patients may reflect senescence of the immune system. $^{21}$

\section{References}

1. Hughes, G.R.V. Systemic lupus erythematosus. Postgrad Med J 1988, 64: 517-521.

2. Ginzler, E.M. \& Schorn, K. Outcome and prognosis in systemic lupus erythematosus. Rheum Clin Dis North Am 1988, 14: 67-78.

3. Wilson, A., Hamilton, M.E., Spyker, D.A. et al. Age influences the clinical and serologic expression of systemic lupus erythematosus. Arthritis Rheum 1981, 24: 1230-1235.

4. Ballou, S.P., Khan, M.A. \& Kushner, I. Clinical features of systemic lupus erythematosus. Differences related to race and age of onset. Arthritis Rheum 1982, 25: 55-60.

5. Catoggio, L.J., Skinner, R.P., Smith, G. \& Maddison, P.J. Systemic lupus erythematosus in the elderly: clinical and serological characteristics. J Rheumatol 1984, 11: 175-181.

6. Maddison, P.J. Systemic lupus erythematosus in the elderly. $J$ Rheumatol 1987, 14(Suppl 13): 182-187.

7. Johnsson, H., Nived, O. \& Sturfelt, G. The effect of age on clinical and serological manifestations in unselected patients with systemic lupus erythematosus. $J$ Rheumatol 1988, 15: 505-509.

8. Foad, B.S., Sheon, R.P. \& Kirsner, A.B. Systemic lupus erythematosus in the elderly. Arch Intern Med 1972, 130: 743-746.

9. Baker, S.B., Rovira, J.R., Campion, E.W. \& Mills, J.A. Late onset systemic lupus erythematosus. Am J Med 1979, 66: 727-732.

10. Font, J., Pallares, L., Cervera, R. et al. Systemic lupus erythematosus in elderly: clinical and serological characteristics. Ann Rheum Dis 1991, 50: 702-705.

11. Urowitz, M.B., Stevens, M.B. \& Shulman, L.E. The influence of age on the clinical pattern of systemic lupus erythematosus. Arthritis Rheum 1967, 10: 319-320.

12. Hochberg, M.C., Boyd, R.E., Ahearn, J.M. et al. Systemic lupus erythematosus: a review of clinico-laboratory features and immunogenetic markers in 150 patients with emphasis on demographic subsets. Medicine 1985, 64: 285-295.
13. Tan, E.M., Cohen, A.S., Fries, J.F. et al. The 1982 revised criteria for the classification of systemic lupus erythematosus. Arthritis Rheum 1982, 25: 1271-1277.

14. Gharavi, A.E., Harris, E.N., Asherson, R.A. \& Hughes, G.R.V. Anticardiolipin antibodies: isotype distribution and phospholipid specificity. Ann Rheum Dis 1987, 46: 1-6.

15. Exner, T., Rickard, K.A. \& Kronenberg, H. Studies on phospholipids in the action of a lupus coagulation inhibitor. Pathology 1975, 7: 319-328.

16. Vianna, J.L., Haga, H.J., Asherson, R.A., Swana, G. \& Hughes, G.R.V. A prospective evaluation of antithyroid antibody prevalence in 100 patients with systemic lupus erythematosus. $J$ Rheumatol 1991, 18: 1193-1195.

17. Manoussakis, M.N., Tzioufas, A.G., Silis, M.G., Pange, P.J.E., Goudevenos, J. \& Moutsopoulos, H.M. High prevalence of anti-cardiolipin and other autoantibodies in a healthy elderly population. Clin Exp Immunol 1987, 69: 557-565.

18. Baer, A.L. \& Pincus, T. Occult systemic lupus erythematosus in elderly men. JAMA 1983, 249: 3350-3352.

19. Bell, D.A. Systemic lupus erythematosus in the elderly. Is it really SLE or systemic Sjögren's syndrome? $J$ Rheumatol 1988, 15: 723-724.

20. Reveille, J.D., Bartollucci, A. \& Alarcon, G.A. Prognosis in systemic lupus erythematosus. Negative impact of increasing age at onset, black race, and thrombocytopenia, as well as causes of death. Arthritis Rheum 1990, 33: 37-48.

21. Stevens, M.B. Connective tissue disease in the elderly. Clin Rheum Dis 1986, 12: 11-32. 